\title{
Embedding Structures Associated with Riordan Arrays and Moment Matrices
}

\author{
Paul Barry \\ School of Science, Waterford Institute of Technology, Waterford, Ireland \\ Correspondence should be addressed to Paul Barry; pbarry@wit.ie \\ Received 17 December 2013; Accepted 14 February 2014; Published 17 March 2014 \\ Academic Editor: Toufik Mansour \\ Copyright (C) 2014 Paul Barry. This is an open access article distributed under the Creative Commons Attribution License, which \\ permits unrestricted use, distribution, and reproduction in any medium, provided the original work is properly cited.
}

Every ordinary Riordan array contains two naturally embedded Riordan arrays. We explore this phenomenon, and we compare it to the situation for certain moment matrices of families of orthogonal polynomials.

\section{Introduction}

Riordan arrays [1] have been used mainly to prove combinatorial identities $[2,3]$. Recently, their links to orthogonal polynomials have been investigated $[4,5]$, while there is a growing literature surrounding their structural properties [610]. In this paper we investigate an embedding structure, common to all ordinary Riordan arrays. We also look at this embedding structure in the context of moment matrices of families of orthogonal polynomials. In addition to some knowledge of Riordan arrays, we assume that the reader has a basic familiarity with the theory of orthogonal polynomials on the real line [11-13], production matrices [14, 15], and continued fractions [16]. We will meet a number of integer sequences and integer triangles in this paper. The On-Line Encyclopedia may be consulted for many of them $[17,18]$. In this paper we will understand by an ordinary Riordan array an integer number triangle whose $(n, k)$-th element $T_{n, k}$ is defined by a pair of power series $g(x)$ and $f(x)$ over the integers with $g(x)=1+g_{1} x+g_{2} x^{2}+\cdots, f(x)=x+f_{2} x^{2}+$ $f_{3} x^{3}+\cdots$, in the following manner:

$$
T_{n, k}=\left[x^{n}\right] g(x) f(x)^{k} .
$$

The group law for Riordan arrays is given by

$$
(g, f) \cdot(h, l)=(g(h \circ f), l \circ f) .
$$

The identity for this law is $I=(1, x)$ and the inverse of $(g, f)$ is $(g, f)^{-1}=(1 /(g \circ \bar{f}), \bar{f})$, where $\bar{f}$ is the compositional inverse of $f$. For a power series $f(x)=\sum_{n=0}^{\infty} a_{n} x^{n}$ with $f(0)=0$, we define the reversion or compositional inverse of $f$ to be the power series $\bar{f}(x)$ such that $f(\bar{f}(x))=x$. We sometimes write this as $\bar{f}=\operatorname{Rev} f$.

If a matrix $A$ is the inverse of the coefficient array of a family of orthogonal polynomials, then we will call it a moment matrix, and we will single out the first column as the moment sequence.

\section{The Canonical Embedding}

Let $(g, f)$ be an ordinary Riordan array $R$, with general term

$$
T_{n, k}=\left[x^{n}\right] g f^{k} .
$$

Then we observe that there are two naturally associated Riordan arrays "embedded" in the array $R$ as follows.

Beginning at the first column of $R$, we take every second column, "raising" the columns appropriately to obtain a lower-triangular matrix $A$. The matrix $A$ is then the Riordan array

$$
A=\left(g, \frac{f^{2}}{x}\right)
$$


with general term $A_{n, k}$ given by

$$
\begin{aligned}
A_{n, k} & =\left[x^{n}\right] g\left(\frac{f^{2}}{x}\right)^{k} \\
& =\left[x^{n}\right] g x^{-k} f^{2 k} \\
& =\left[x^{n+k}\right] g f^{2 k} \\
& =T_{n+k, 2 k} .
\end{aligned}
$$

Similarly, starting at the second column of $R$, taking every second column and "raising" all columns appropriately to obtain a lower-triangular matrix, we obtain a matrix $B$. This matrix $B$ is then a Riordan array, given by

$$
B=\left(g \frac{f}{x}, \frac{f^{2}}{x}\right) .
$$

We have

$$
B=\left(\frac{f}{x}, x\right) \cdot A
$$

The general term $B_{n, k}$ of $B$ is given by

$$
\begin{aligned}
B_{n, k} & =\left[x^{n}\right] g \frac{f}{x}\left(\frac{f^{2}}{x}\right)^{k} \\
& =\left[x^{n}\right] g x^{-k-1} f^{2 k+1} \\
& =\left[x^{n+k+1}\right] g f^{2 k+1} \\
& =T_{n+k+1,2 k+1} .
\end{aligned}
$$

Example 1. We take the example of the binomial matrix

$$
R=\left(\frac{1}{1-x}, \frac{x}{1-x}\right)
$$

We then have

$$
\begin{array}{r}
A=\left(\frac{1}{1-x}, \frac{x}{(1-x)^{2}}\right) \\
\text { with general term }\left(\begin{array}{c}
n+k \\
2 k
\end{array}\right), \\
B=\left(\frac{1}{(1-x)^{2}}, \frac{x}{(1-x)^{2}}\right) \\
\text { with general term }\left(\begin{array}{c}
n+k+1 \\
2 k+1
\end{array}\right) .
\end{array}
$$

The following decomposition makes this clear:

$$
\left(\begin{array}{ccccccc}
1 & 0 & 0 & 0 & 0 & 0 & \cdots \\
1 & 1 & 0 & 0 & 0 & 0 & \cdots \\
1 & 2 & 1 & 0 & 0 & 0 & \cdots \\
1 & 3 & 3 & 1 & 0 & 0 & \cdots \\
1 & 4 & 6 & 4 & 1 & 0 & \cdots \\
1 & 5 & 10 & 10 & 5 & 1 & \cdots \\
\vdots & \vdots & \vdots & \vdots & \vdots & \vdots & \ddots
\end{array}\right)
$$

The matrices $A$ and $B$ are the coefficient arrays of the MorganVoyce polynomials $b_{n}(x)$ and $B_{n}(x)$, respectively.

Example 2. We take the Riordan array

$$
R=(c(x), x c(x))
$$

where

$$
c(x)=\frac{1-\sqrt{1-4 x}}{2 x} .
$$

Then we find that

$$
A=\left(c(x), x c(x)^{2}\right), \quad B=\left(c(x)^{2}, x c(x)^{2}\right) .
$$

The matrix $R$ begins with

$$
\left(\begin{array}{ccccccc}
1 & 0 & 0 & 0 & 0 & 0 & \cdots \\
1 & 1 & 0 & 0 & 0 & 0 & \cdots \\
2 & 2 & 1 & 0 & 0 & 0 & \cdots \\
5 & 5 & 3 & 1 & 0 & 0 & \cdots \\
14 & 14 & 9 & 4 & 1 & 0 & \cdots \\
42 & 42 & 28 & 14 & 5 & 1 & \cdots \\
\vdots & \vdots & \vdots & \vdots & \vdots & \vdots & \ddots
\end{array}\right)
$$

We note that the matrix $A=\left(c(x), x c(x)^{2}\right)$ is the moment array for the family of orthogonal polynomials with coefficient array given by

$$
A^{-1}=\left(c(x), x c(x)^{2}\right)^{-1}=\left(\frac{1}{1+x}, \frac{x}{(1+x)^{2}}\right) \text {. }
$$

Denoting this family by $P_{n}(x)$, we have

$$
P_{n}(x)=(x-2) P_{n-1}(x)-P_{n-2}(x),
$$

with $P_{0}(x)=1$ and $P_{1}(x)=x-1$. Similarly the matrix $B=\left(c(x)^{2}, x c(x)^{2}\right)$ is the moment array for the family of orthogonal polynomials with coefficient array given by

$$
B^{-1}=\left(\frac{1}{(1+x)^{2}}, \frac{x}{(1+x)^{2}}\right) \text {. }
$$

Denoting this family by $Q_{n}(x)$, we have

$$
Q_{n}(x)=(x-2) Q_{n-1}(x)-Q_{n-2}(x),
$$

with $Q_{0}(x)=1$ and $Q_{1}(x)=x-2$.

The inverse matrix $R^{-1}$ is given by

$$
\left(\begin{array}{ccccccc}
1 & 0 & 0 & 0 & 0 & 0 & \cdots \\
-1 & 1 & 0 & 0 & 0 & 0 & \cdots \\
0 & -2 & 1 & 0 & 0 & 0 & \cdots \\
0 & 1 & -3 & 1 & 0 & 0 & \cdots \\
0 & 0 & 3 & -4 & 1 & 0 & \cdots \\
0 & 0 & -1 & 6 & -5 & 1 & \cdots \\
\vdots & \vdots & \vdots & \vdots & \vdots & \vdots & \ddots
\end{array}\right)
$$

which is the Riordan array $(1-x, x(1-x))$. In it we see the elements of $A^{-1}$ and $B^{-1}$ in staggered fashion. 


\section{A Counter Example}

It is natural to ask the question: is a matrix that contains two embedded Riordan arrays as above itself a Riordan array? The following example shows that this is not a sufficient condition on an array to be Riordan.

Example 3. We will construct an invertible integer lowertriangular matrix which has two embedded Riordan arrays in the fashion above, but which is not itself a Riordan array. We start with the essentially two-period sequence $\left(a_{n}\right)_{n \geq 0}$

$$
1,2,3,2,3,2,3, \ldots
$$

We form the matrix

$$
\left(\begin{array}{ccccccc}
1 & 0 & 0 & 0 & 0 & 0 & \cdots \\
-2 & 1 & 0 & 0 & 0 & 0 & \cdots \\
0 & -3 & 1 & 0 & 0 & 0 & \cdots \\
0 & 0 & -2 & 1 & 0 & 0 & \cdots \\
0 & 0 & 0 & -3 & 1 & 0 & \cdots \\
0 & 0 & 0 & 0 & -2 & 1 & \cdots \\
\vdots & \vdots & \vdots & \vdots & \vdots & \vdots & \ddots
\end{array}\right)
$$

The inverse of this matrix begins with

$$
\left(\begin{array}{ccccccc}
1 & 0 & 0 & 0 & 0 & 0 & \cdots \\
2 & 1 & 0 & 0 & 0 & 0 & \cdots \\
6 & 3 & 1 & 0 & 0 & 0 & \cdots \\
12 & 6 & 2 & 1 & 0 & 0 & \cdots \\
36 & 18 & 6 & 3 & 1 & 0 & \cdots \\
72 & 36 & 12 & 6 & 2 & 1 & \cdots \\
\vdots & \vdots & \vdots & \vdots & \vdots & \vdots & \ddots
\end{array}\right)
$$

where we note an alternating pattern of constant columns (with generating functions $(1+2 x) /\left(1-6 x^{2}\right)$ and $(1+3 x) /(1-$ $\left.6 x^{2}\right)$, resp.). Removing the first row of this matrix provides us with a production matrix, which is not of the form that produces a Riordan array (after the first column, subsequent columns would be shifted versions of the second column $[14,15])$. Thus the resulting matrix will not be a Riordan array. This resulting matrix begins with

$$
\left(\begin{array}{ccccccc}
1 & 0 & 0 & 0 & 0 & 0 & \cdots \\
2 & 1 & 0 & 0 & 0 & 0 & \cdots \\
10 & 5 & 1 & 0 & 0 & 0 & \cdots \\
62 & 31 & 7 & 1 & 0 & 0 & \cdots \\
430 & 215 & 51 & 10 & 1 & 0 & \cdots \\
3194 & 1597 & 389 & 87 & 12 & 1 & \cdots \\
\vdots & \vdots & \vdots & \vdots & \vdots & \vdots & \ddots
\end{array}\right) .
$$

We now observe that for this matrix, we have

$$
\begin{aligned}
& A=\left(\frac{1}{1+2 x}, \frac{x}{1+5 x+6 x^{2}}\right)^{-1}, \\
& B=\left(\frac{1}{1+5 x+6 x^{2}}, \frac{x}{1+5 x+6 x^{2}}\right)^{-1} .
\end{aligned}
$$

We notice that the sequence

$$
1,2,10,62,430,3194, \ldots
$$

has generating function given by the continued fraction

$$
\frac{1}{1-\frac{2 x}{1-\frac{3 x}{1-\frac{2 x}{1-\cdots}}}}
$$

and secondly that

$$
1+5 x+6 x^{2}=1+(2+3) x+2 \cdot 3 x^{2}=(1+2 x)(1+3 x) .
$$

This construction is easily generalized.

\section{Embedding a Riordan Array}

Another natural question to ask is: if we are given a Riordan array $A$, is it possible to embed it as above into a Riordan array $R$ ? For this, we let

$$
A=(u, v),
$$

and seek to determine

$$
R=(g, f)
$$

such that $A$ embeds into $R$. For this, we need

$$
u=g, \quad v=\frac{f^{2}}{x} .
$$

Thus we require that

$$
f=\sqrt{x v}=x \sqrt{\frac{v}{x}}
$$

Since we are working in the context of integer valued Riordan arrays, we require that $\sqrt{v / x}$ generates an integer sequence. We can state our result as follows.

Proposition 4. The Riordan array

$$
A=(u, v)
$$

can be embedded in the Riordan array

$$
R=\left(g, x \sqrt{\frac{v}{x}}\right)
$$

on condition that $\sqrt{v / x}$ is the generating function of an integer sequence.

Example 5. The Riordan array

$$
A=\left(\frac{1}{\sqrt{1-4 x}}, \frac{x}{1-4 x}\right)
$$


can be embedded in the Riordan array

$$
R=\left(\frac{1}{\sqrt{1-4 x}}, \frac{x}{\sqrt{1-4 x}}\right) .
$$

For this example, the matrix $A$ begins with

$$
\left(\begin{array}{ccccccc}
1 & 0 & 0 & 0 & 0 & 0 & \cdots \\
2 & 1 & 0 & 0 & 0 & 0 & \cdots \\
6 & 6 & 1 & 0 & 0 & 0 & \cdots \\
20 & 30 & 10 & 1 & 0 & 0 & \cdots \\
70 & 140 & 70 & 14 & 1 & 0 & \cdots \\
252 & 630 & 420 & 126 & 18 & 1 & \cdots \\
\vdots & \vdots & \vdots & \vdots & \vdots & \vdots & \ddots
\end{array}\right)
$$

while $R$ begins with

$$
\left(\begin{array}{ccccccc}
1 & 0 & 0 & 0 & 0 & 0 & \cdots \\
2 & 1 & 0 & 0 & 0 & 0 & \cdots \\
6 & 4 & 1 & 0 & 0 & 0 & \cdots \\
20 & 16 & 6 & 1 & 0 & 0 & \cdots \\
70 & 64 & 30 & 8 & 1 & 0 & \cdots \\
252 & 256 & 140 & 48 & 10 & 1 & \cdots \\
\vdots & \vdots & \vdots & \vdots & \vdots & \vdots & \ddots
\end{array}\right) .
$$

\section{A Cascading Decomposition}

We note that we can "cascade" this embedding process, in the sense that, given a Riordan array $R$, with embedded Riordan arrays $A$ and $B$, we can consider decomposing $A$ and $B$ in their turns and then continue this process. For instance, we can decompose

$$
A=\left(g, \frac{f^{2}}{x}\right)
$$

into the two matrices

$$
A_{A}=\left(g, \frac{f^{4}}{x^{3}}\right), \quad B_{A}=\left(g \frac{f^{2}}{x^{2}}, \frac{f^{4}}{x^{3}}\right) .
$$

In their turn $A_{A}$ and $B_{A}$ can be decomposed and so on.

\section{Embedding and Orthogonal Polynomials}

The phenomenon of embedding as described above is not confined to Riordan arrays, as the continued fraction example above shows. To further amplify this point, we give another example involving a continued fraction. Although we take a particular case, the general case can be inferred easily from it. Thus we take the particular case of the continued fraction

$$
\frac{1}{1-\frac{2 x}{1-\frac{3 x}{1-\frac{5 x}{1-\frac{2 x}{1-\frac{5 x}{1-\cdots}}}}}}
$$

This continued fraction is equal to

$$
1-2 x-\frac{1}{1-8 x-\frac{6 x^{2}}{1-5 x-\frac{10 x^{2}}{1-7 x-\frac{15 x^{2}}{1-8 x-\frac{10 x^{2}}{1-5 x-\cdots}}}}}
$$

By the theory of orthogonal polynomials, the power series expressed by both continued fractions is the generating function for the moment sequence of the family of orthogonal polynomials whose moment matrix (the inverse of the coefficient array of the orthogonal polynomials) has production matrix given by

$$
\left(\begin{array}{ccccccc}
2 & 1 & 0 & 0 & 0 & 0 & \cdots \\
6 & 8 & 1 & 0 & 0 & 0 & \cdots \\
0 & 10 & 5 & 1 & 0 & 0 & \cdots \\
0 & 0 & 15 & 7 & 1 & 0 & \cdots \\
0 & 0 & 0 & 6 & 8 & 1 & \cdots \\
0 & 0 & 0 & 0 & 10 & 5 & \cdots \\
\vdots & \vdots & \vdots & \vdots & \vdots & \vdots & \ddots
\end{array}\right)
$$

This production matrix generates the moment matrix $A$ that begins with

$$
\left(\begin{array}{ccccccc}
1 & 0 & 0 & 0 & 0 & 0 & \cdots \\
2 & 1 & 0 & 0 & 0 & 0 & \cdots \\
10 & 10 & 1 & 0 & 0 & 0 & \cdots \\
80 & 100 & 15 & 1 & 0 & 0 & \cdots \\
760 & 1030 & 190 & 22 & 1 & 0 & \cdots \\
7700 & 10900 & 2310 & 350 & 30 & 1 & \cdots \\
\vdots & \vdots & \vdots & \vdots & \vdots & \vdots & \ddots
\end{array}\right)
$$

In order to produce an embedding for this matrix, we proceed as follows. We form the matrix

$$
\left(\begin{array}{ccccccc}
1 & 0 & 0 & 0 & 0 & 0 & \cdots \\
-2 & 1 & 0 & 0 & 0 & 0 & \cdots \\
0 & -3 & 1 & 0 & 0 & 0 & \cdots \\
0 & 0 & -5 & 1 & 0 & 0 & \cdots \\
0 & 0 & 0 & -2 & 1 & 0 & \cdots \\
0 & 0 & 0 & 0 & -3 & 1 & \cdots \\
\vdots & \vdots & \vdots & \vdots & \vdots & \vdots & \ddots
\end{array}\right)
$$

We invert this matrix, remove the first row of the resulting matrix, and use this new matrix as a production matrix. The generated matrix $R$ then begins with

$$
\left(\begin{array}{ccccccc}
1 & 0 & 0 & 0 & 0 & 0 & \cdots \\
2 & 1 & 0 & 0 & 0 & 0 & \cdots \\
10 & 5 & 1 & 0 & 0 & 0 & \cdots \\
80 & 40 & 10 & 1 & 0 & 0 & \cdots \\
760 & 380 & 100 & 12 & 1 & 0 & \cdots \\
7700 & 3850 & 1030 & 130 & 15 & 1 & \cdots \\
\vdots & \vdots & \vdots & \vdots & \vdots & \vdots & \ddots
\end{array}\right) .
$$


The moment matrix $A$ is evidently embedded in the matrix $R$. We can show that the corresponding matrix $B$ is the moment matrix for the family of orthogonal polynomials whose moments have generating function given by

$$
\frac{1}{1-5 x-\frac{15 x^{2}}{1-7 x-\frac{6 x^{2}}{1-8 x-\frac{10 x^{2}}{1-5 x-\cdots}}}}
$$

The matrix $B$ begins with

$$
\left(\begin{array}{ccccccc}
1 & 0 & 0 & 0 & 0 & 0 & \cdots \\
5 & 1 & 0 & 0 & 0 & 0 & \cdots \\
40 & 12 & 1 & 0 & 0 & 0 & \cdots \\
380 & 130 & 20 & 1 & 0 & 0 & \cdots \\
3850 & 1410 & 300 & 25 & 1 & 0 & \cdots \\
40400 & 15520 & 4060 & 440 & 32 & 1 & \cdots \\
\vdots & \vdots & \vdots & \vdots & \vdots & \vdots & \ddots
\end{array}\right)
$$

and it has production matrix

$$
\left(\begin{array}{ccccccc}
5 & 1 & 0 & 0 & 0 & 0 & \cdots \\
15 & 7 & 1 & 0 & 0 & 0 & \cdots \\
0 & 6 & 8 & 1 & 0 & 0 & \cdots \\
0 & 0 & 10 & 5 & 1 & 0 & \cdots \\
0 & 0 & 0 & 15 & 7 & 1 & \cdots \\
0 & 0 & 0 & 0 & 6 & 8 & \cdots \\
\vdots & \vdots & \vdots & \vdots & \vdots & \vdots & \ddots
\end{array}\right)
$$

The matrix $R^{-1}$ can now be characterized as the coefficient array of a family of polynomials $R_{n}(x)$ defined as follows. We let $P_{n}(x)$ be the family of orthogonal polynomials with coefficient array $A^{-1}$, and we let $Q_{n}(x)$ be the family of orthogonal polynomials with coefficient $B^{-1}$. Then we have

$$
R_{n}(x)= \begin{cases}Q_{n / 2}(x) x^{n / 2}, & \text { if } n \text { is even; } \\ P_{\lceil n / 2\rceil}(x) x^{\lfloor n / 2\rfloor}, & \text { otherwise. }\end{cases}
$$

In the general case of a moment sequence generated by the continued fraction

$$
\frac{1}{1-\frac{\alpha x}{1-\frac{\beta x}{1-\frac{\gamma x}{1-\frac{\alpha x}{1-\cdots}}}}},
$$

the matrix $A$ will be generated by the production matrix

$$
\left(\begin{array}{ccccccc}
\alpha & 1 & 0 & 0 & 0 & 0 & \cdots \\
\alpha \beta & \beta+\gamma & 1 & 0 & 0 & 0 & \cdots \\
0 & \alpha \gamma & \alpha+\beta & 1 & 0 & 0 & \cdots \\
0 & 0 & \beta \gamma & \alpha+\gamma & 1 & 0 & \cdots \\
0 & 0 & 0 & \alpha \beta & \beta+\gamma & 1 & \cdots \\
0 & 0 & 0 & 0 & \alpha \gamma & \alpha+\beta & \cdots \\
\vdots & \vdots & \vdots & \vdots & \vdots & \vdots & \ddots
\end{array}\right),
$$

while the matrix $B$ is generated by the production matrix

$$
\left(\begin{array}{ccccccc}
\alpha+\beta & 1 & 0 & 0 & 0 & 0 & \cdots \\
\beta \gamma & \alpha+\gamma & 1 & 0 & 0 & 0 & \cdots \\
0 & \alpha \beta & \beta+\gamma & 1 & 0 & 0 & \cdots \\
0 & 0 & \alpha \gamma & \alpha+\beta & 1 & 0 & \cdots \\
0 & 0 & 0 & \beta \gamma & \alpha+\gamma & 1 & \cdots \\
0 & 0 & 0 & 0 & \alpha \beta & \beta+\gamma & \cdots \\
\vdots & \vdots & \vdots & \vdots & \vdots & \vdots & \ddots
\end{array}\right) .
$$

\section{Embedding, Orthogonal Polynomials, and Riordan Arrays}

In this section, we consider the case of two related families of orthogonal polynomials $P_{n}(x)$ and $Q_{n}(x)$ defined by

$$
P_{n}(x)=(x-7) P_{n-1}(x)-12 P_{n-2},
$$

with $P_{0}(x)=1, P_{1}(x)=x-3$, and

$$
Q_{n}(x)=(x-7) Q_{n-1}(x)-12 Q_{n-2},
$$

with $Q_{0}(x)=1, Q_{1}(x)=x-7$. We note that

$$
(1+3 x)(1+4 x)=1+7 x+12 x^{2} .
$$

The coefficient array of the polynomials $P_{n}(x)$ is then given by the Riordan array

$$
A^{-1}=\left(\frac{1}{1+3 x}, \frac{x}{1+7 x+12 x^{2}}\right),
$$

while that of $Q_{n}(x)$ is given by

$$
B^{-1}=\left(\frac{1}{1+7 x+12 x^{2}}, \frac{x}{1+7 x+12 x^{2}}\right) .
$$

The moment matrix $A$ begins with

$$
\left(\begin{array}{ccccccc}
1 & 0 & 0 & 0 & 0 & 0 & \cdots \\
3 & 1 & 0 & 0 & 0 & 0 & \cdots \\
21 & 10 & 1 & 0 & 0 & 0 & \cdots \\
183 & 103 & 17 & 1 & 0 & 0 & \cdots \\
1785 & 1108 & 234 & 24 & 1 & 0 & \cdots \\
18651 & 12349 & 3034 & 414 & 31 & 1 & \cdots \\
\vdots & \vdots & \vdots & \vdots & \vdots & \vdots & \ddots
\end{array}\right)
$$

while the moment matrix $B$ starts with

$$
\left(\begin{array}{ccccccc}
1 & 0 & 0 & 0 & 0 & 0 & \cdots \\
7 & 1 & 0 & 0 & 0 & 0 & \cdots \\
61 & 14 & 1 & 0 & 0 & 0 & \cdots \\
595 & 171 & 21 & 1 & 0 & 0 & \cdots \\
6217 & 2044 & 330 & 28 & 1 & 0 & \cdots \\
68047 & 24485 & 4690 & 538 & 35 & 1 & \cdots \\
\vdots & \vdots & \vdots & \vdots & \vdots & \vdots & \ddots
\end{array}\right) .
$$

Letting

$$
R_{n}(x)= \begin{cases}Q_{n / 2}(x) x^{n / 2}, & \text { if } n \text { is even; } \\ P_{(n+1) / 2}(x) x^{\lfloor n / 2\rfloor}, & \text { otherwise }\end{cases}
$$


we find that the inverse $R$ of the coefficient array of the family $R_{n}(x)$ is given by

$$
\left(\begin{array}{ccccccc}
1 & 0 & 0 & 0 & 0 & 0 & \cdots \\
3 & 1 & 0 & 0 & 0 & 0 & \cdots \\
21 & 7 & 1 & 0 & 0 & 0 & \cdots \\
183 & 61 & 10 & 1 & 0 & 0 & \cdots \\
1785 & 595 & 103 & 14 & 1 & 0 & \cdots \\
18651 & 6217 & 1108 & 171 & 17 & 1 & \cdots \\
\vdots & \vdots & \vdots & \vdots & \vdots & \vdots & \ddots
\end{array}\right)
$$

Thus the two Riordan arrays $A$ and $B$, which are the moment arrays of the two families of orthogonal polynomials $P_{n}(x)$ and $Q_{n}(x)$, respectively, embed into the generalized moment array $R$ for the family of polynomials $R_{n}(x)$. Now the production matrix of $R$ begins with

$$
\left(\begin{array}{ccccccc}
3 & 1 & 0 & 0 & 0 & 0 & \cdots \\
12 & 4 & 1 & 0 & 0 & 0 & \cdots \\
36 & 12 & 3 & 1 & 0 & 0 & \cdots \\
144 & 48 & 12 & 4 & 1 & 0 & \cdots \\
432 & 144 & 36 & 12 & 3 & 1 & \cdots \\
1728 & 576 & 144 & 48 & 12 & 4 & \cdots \\
\vdots & \vdots & \vdots & \vdots & \vdots & \vdots & \ddots
\end{array}\right)
$$

where the columns have generating functions $(1+4 x) /(1-$ $\left.12 x^{2}\right),(1+3) /\left(1-12 x^{2}\right)$, respectively. We now observe that this production matrix is obtained by removing the first row of the inverse of the matrix

$$
\left(\begin{array}{ccccccc}
1 & 0 & 0 & 0 & 0 & 0 & \cdots \\
-3 & 1 & 0 & 0 & 0 & 0 & \cdots \\
0 & -4 & 1 & 0 & 0 & 0 & \cdots \\
0 & 0 & -3 & 1 & 0 & 0 & \cdots \\
0 & 0 & 0 & -4 & 1 & 0 & \cdots \\
0 & 0 & 0 & 0 & -3 & 1 & \cdots \\
\vdots & \vdots & \vdots & \vdots & \vdots & \vdots & \ddots
\end{array}\right)
$$

We note finally that the sequence

$$
1,3,21,183,1785,18651,204141, \ldots
$$

has generating function given by

$$
g(x)=\frac{1}{1-\frac{3 x}{1-\frac{4 x}{1-\frac{3 x}{1-\cdots}}}} .
$$

We have in fact that

$$
g(x)=\frac{1}{x} \operatorname{Rev} \frac{x(1-4 x)}{1-x} .
$$

On the other hand, if we let

$$
P_{n}(x)=(x-7) P_{n-1}(x)-12 P_{n-2}(x)
$$

but this time take $P_{0}(x)=1$ and $P_{1}(x)=x-4$, then the matrix $A$ becomes

$$
A=\left(\frac{1}{1+4 x}, \frac{x}{1+7 x+12 x^{2}}\right)^{-1} .
$$

The matrix $A$ then begins with

$$
\left(\begin{array}{ccccccc}
1 & 0 & 0 & 0 & 0 & 0 & \cdots \\
4 & 1 & 0 & 0 & 0 & 0 & \cdots \\
28 & 11 & 1 & 0 & 0 & 0 & \cdots \\
244 & 117 & 18 & 1 & 0 & 0 & \cdots \\
2380 & 1279 & 255 & 25 & 1 & 0 & \cdots \\
24868 & 14393 & 3364 & 442 & 32 & 1 & \cdots \\
\vdots & \vdots & \vdots & \vdots & \vdots & \vdots & \ddots
\end{array}\right)
$$

where the moment sequence

$$
1,4,28,244,2380, \ldots
$$

has generating function

$$
\frac{1}{1-\frac{4 x}{1-\frac{3 x}{1-\frac{4 x}{1-\cdots}}}}
$$

or equivalently

$$
\frac{1}{1-4 x-\frac{12 x^{2}}{1-7 x-\frac{12 x^{2}}{1-7 x-\cdots}}}
$$

In this case, the matrix $R$ begins with

$$
\left(\begin{array}{ccccccc}
1 & 0 & 0 & 0 & 0 & 0 & \cdots \\
4 & 1 & 0 & 0 & 0 & 0 & \cdots \\
28 & 7 & 1 & 0 & 0 & 0 & \cdots \\
244 & 61 & 11 & 1 & 0 & 0 & \cdots \\
2380 & 595 & 117 & 14 & 1 & 0 & \cdots \\
24868 & 6217 & 1279 & 171 & 18 & 1 & \cdots \\
\vdots & \vdots & \vdots & \vdots & \vdots & \vdots & \ddots
\end{array}\right)
$$

This matrix is then associated with the matrix

$$
\left(\begin{array}{ccccccc}
1 & 0 & 0 & 0 & 0 & 0 & \cdots \\
-4 & 1 & 0 & 0 & 0 & 0 & \cdots \\
0 & -3 & 1 & 0 & 0 & 0 & \cdots \\
0 & 0 & -4 & 1 & 0 & 0 & \cdots \\
0 & 0 & 0 & -3 & 1 & 0 & \cdots \\
0 & 0 & 0 & 0 & -4 & 1 & \cdots \\
\vdots & \vdots & \vdots & \vdots & \vdots & \vdots & \ddots
\end{array}\right)
$$

\section{Conflict of Interests}

The author declares that there is no conflict of interests regarding the publication of this paper. 


\section{References}

[1] L. W. Shapiro, S. Getu, W.-J. Woan, and L. C. Woodson, “The Riordan group," Discrete Applied Mathematics, vol. 34, no. 1-3, pp. 229-239, 1991.

[2] C. Corsani, D. Merlini, and R. Sprugnoli, "Left-inversion of combinatorial sums," Discrete Mathematics, vol. 180, no. 1-3, pp. 107-122, 1998.

[3] R. Sprugnoli, "Riordan arrays and combinatorial sums," Discrete Mathematics, vol. 132, no. 1-3, pp. 267-290, 1994.

[4] P. Barry and A. Hennessy, "Meixner-type results for Riordan arrays and associated integer sequences," Journal of Integer Sequences, vol. 13, no. 9, article 10.9.4, 2010.

[5] P. Barry, "Riordan arrays, orthogonal polynomials as moments, and Hankel transforms," Journal of Integer Sequences, vol. 14, no. 2, article 11.2.2, 2011.

[6] G.-S. Cheon, H. Kim, and L. W. Shapiro, "Riordan group involutions," Linear Algebra and Its Applications, vol. 428, no. 4, pp. 941-952, 2008.

[7] D. E. Davenport, L. W. Shapiro, and L. C. Woodson, "The double Riordan group," The Electronic Journal of Combinatorics, vol. 18, no. 2, Paper 33, 2012.

[8] T.-X. He and R. Sprugnoli, "Sequence characterization of Riordan arrays," Discrete Mathematics, vol. 309, no. 12, pp. 39623974, 2009.

[9] S.-T. Jin, "A characterization of the Riordan Bell subgroup by Csequences," The Korean Journal of Mathematics, vol. 17, no. 2, pp. 147-154, 2009.

[10] D. Merlini, D. G. Rogers, R. Sprugnoli, and M. C. Verri, "On some alternative characterizations of Riordan arrays," Canadian Journal of Mathematics, vol. 49, no. 2, pp. 301-320, 1997.

[11] T. S. Chihara, An Introduction to Orthogonal Polynomials, Gordon and Breach, New York, NY, USA, 1978.

[12] W. Gautschi, Orthogonal Polynomials: Computation and Approximation, Clarendon Press, Oxford, UK, 2004.

[13] G. Szegö, Orthogonal Polynomials, American Mathematical Society, Providence, RI, USA, 4th edition, 1975.

[14] E. Deutsch, L. Ferrari, and S. Rinaldi, "Production matrices," Advances in Applied Mathematics, vol. 34, no. 1, pp. 101-122, 2005.

[15] E. Deutsch, L. Ferrari, and S. Rinaldi, "Production matrices and Riordan arrays," Annals of Combinatorics, vol. 13, no. 1, pp. 6585, 2009.

[16] H. S. Wall, Analytic Theory of Continued Fractions, AMS Chelsea, New York, NY, USA, 1967.

[17] N. J. A. Sloane, "The On-Line Encyclopedia of Integer Sequences," 2011, http://oeis.org.

[18] N. J. A. Sloane, "The on-line Encyclopedia of Integer Sequences," Notices of the American Mathematical Society, vol. 50, no. 8, pp. 912-915, 2003. 


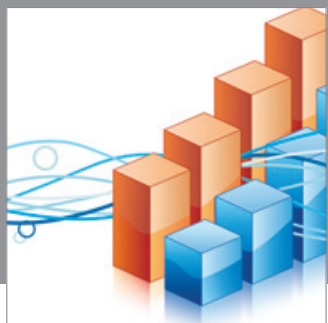

Advances in

Operations Research

mansans

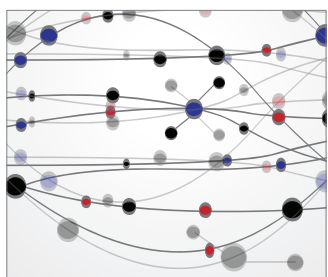

The Scientific World Journal
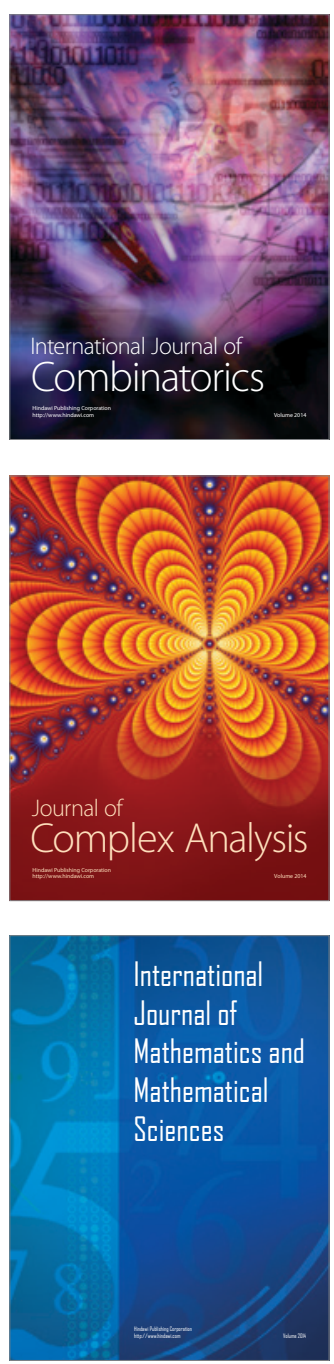
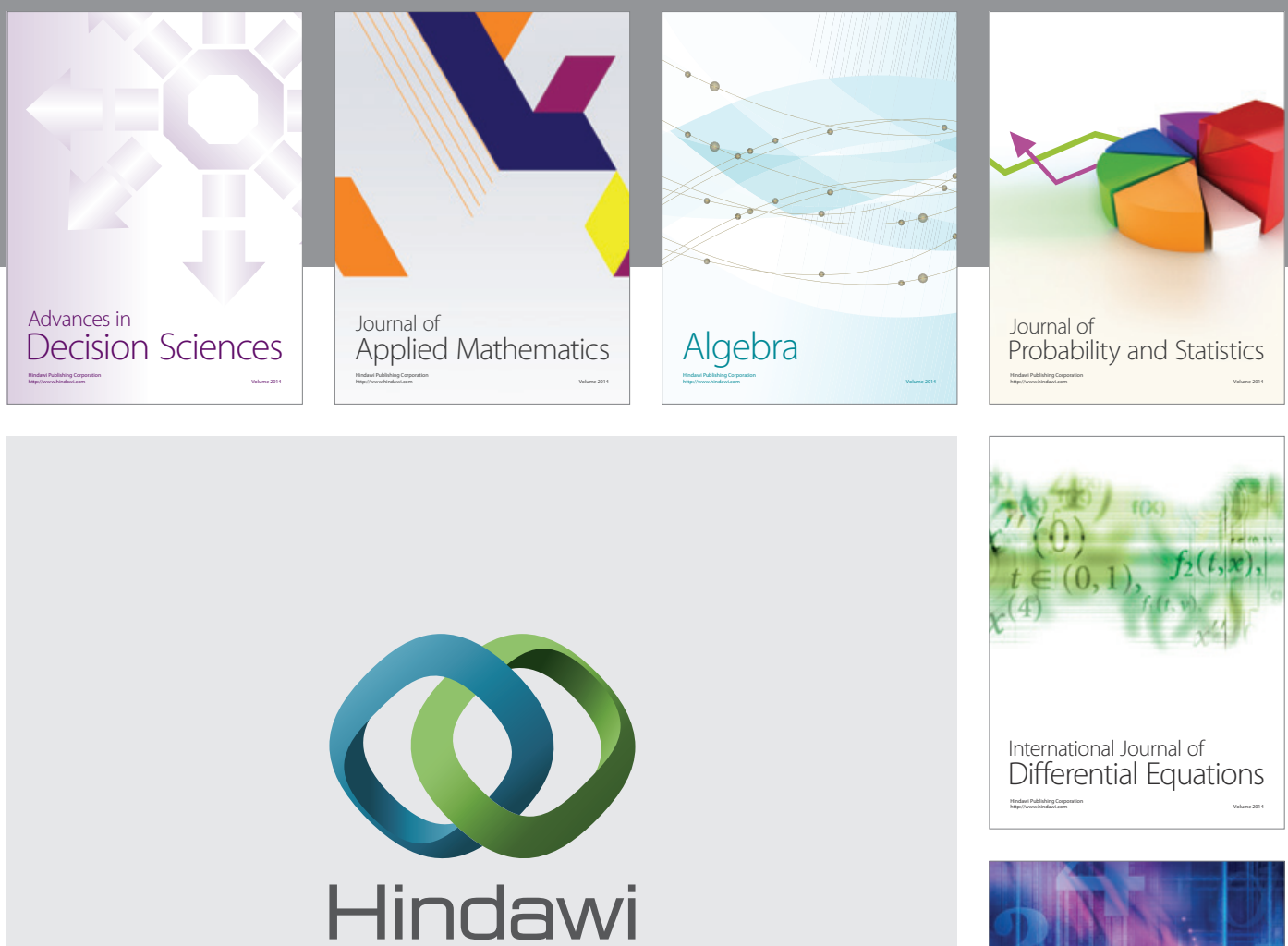

Submit your manuscripts at http://www.hindawi.com
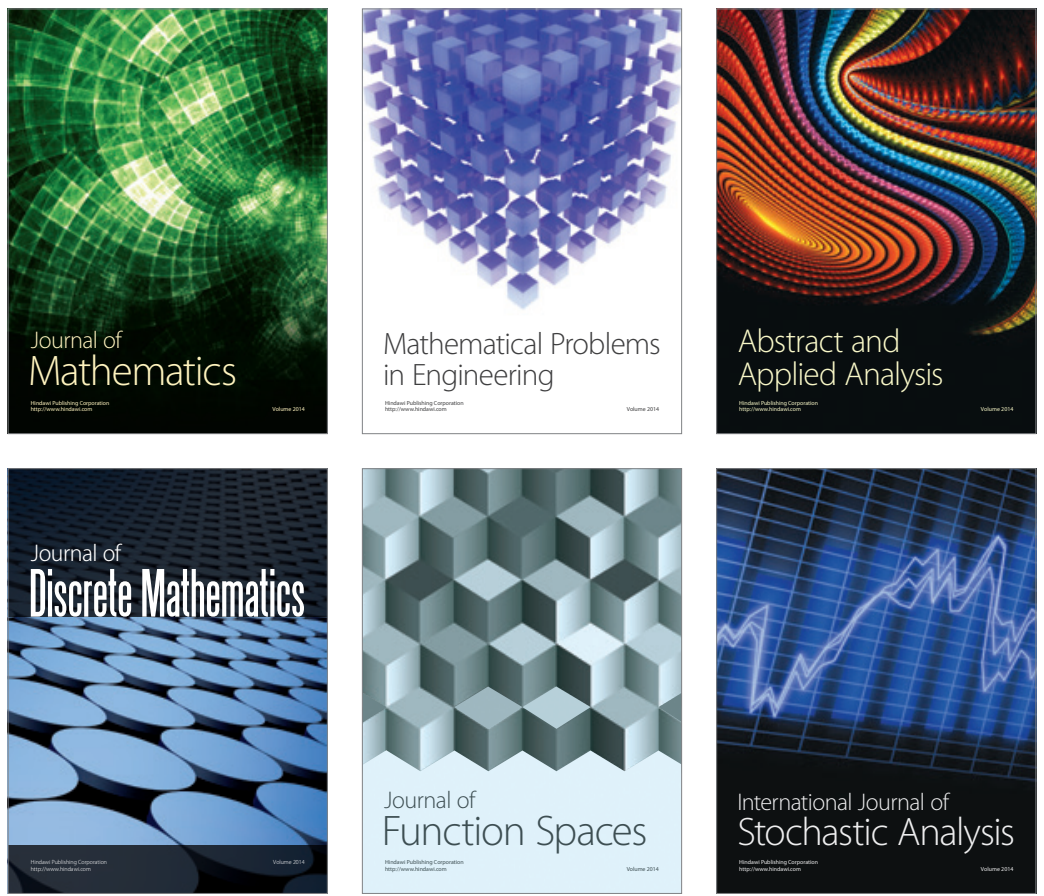

Journal of

Function Spaces

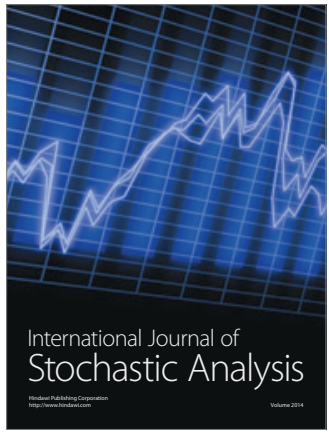

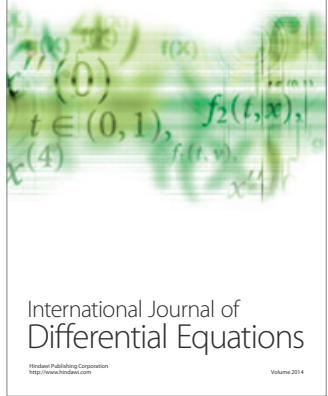
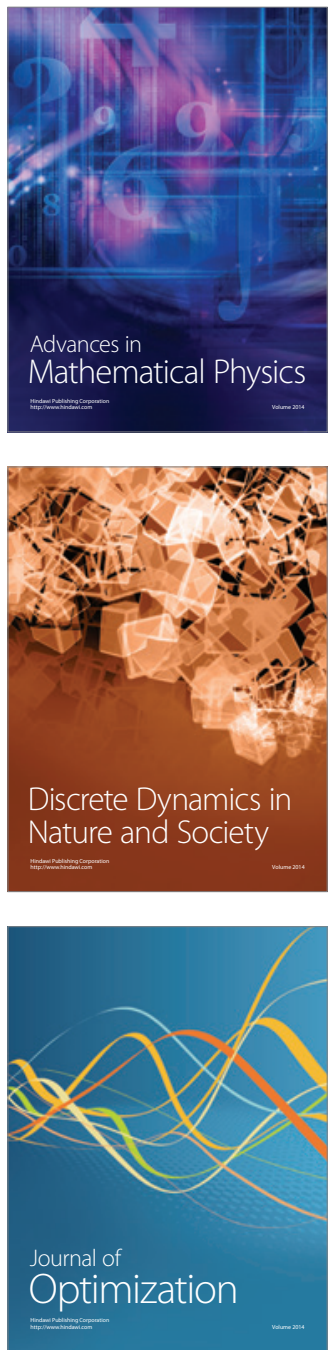